\title{
BEST PRACTICES FOR HAND HYGIENE COMPLIANCE BY HEALTH CARE PROVIDERS IN THE INPATIENT SETTING
}

\author{
Cynthia C. Akpaka \\ Medical University of South Carolina, Charleston, South Carolina, USA
}

Received 2014-04-03; Revised 2014-05-08; Accepted 2014-05-14

\begin{abstract}
This study discusses the strategies that have proven successful in improving adherence to hand hygiene policies among health care providers working in acute care, inpatient settings. Review of literature suggests that while some efforts to improve hand hygiene compliance have focused on environmental engineering other efforts have focused mostly on broad-based educational and motivational initiatives, such as online educational programs and staff training. Both environmental engineering and educational interventions have resulted in improvements in compliance rates, ranging from 25.4 to $97 \%$. Hospitals and healthcare facilities that already use these strategies should continue to do so while evaluating factors that may lead to further improvements. Hospitals and facilities that do not already have these strategies in place should adopt them to help curb the transmission of microbes. These strategies may include random audits, well-written protocols, visual reminders, training for new staff and regular continuing education for current staff.
\end{abstract}

Keywords: Hand Hygiene, Hand-Washing, Best Practices, Adherence, Quality Improvement Initiatives

\section{INTRODUCTION}

Nosocomial infection causes high patient morbidity, increased length of stay and high care cost and thus strategies to reduce transmission and spread of nosocomial infection decrease cost and help with resource utilization (Graves, 2004). A key component of infection control measures is ensuring adequate hand washing by health care providers using appropriate techniques and solvents. Hand washing is the single most important strategy to prevent infections acquired in the hospital Centers for Disease Control (CDCP, 2011). The CDCP (2011) reported several factors that contribute to poor hand washing compliance among health care providers: Lack of knowledge among health care providers about how hands become contaminated and how hand hygiene reduces the spread of infection, lack of understanding of the correct hand hygiene technique, understaffing and overcrowding, poor access to hand washing facilities, irritant contact dermatitis associated with frequent exposure to soap and water and lack of institutional commitment to good hand hygiene. This study includes a review of the strategies that have proven successful in overcoming these barriers and improving adherence to hand hygiene policies among health care providers working in inpatient setting.

According to Graves (2004), about 1 in 10 hospitalized patients will become infected after their admission to the hospital that will results in extended hospital stay, during which time patients will occupy limited hospital beds and require additional assessments and procedures. Preventing infection saves these scarce resources and for some of these resources, the associated expenditures may be eliminated. These savings could be expressed in terms of saving on drugs and other products patients need (Graves, 2004). In addition to creating cost-savings, infection control reduces patient suffering that result from extended hospital stays. Because hand washing is considered an important procedure to prevent hospital-acquired infection, exploring strategies to improve the hand washing compliance of health care workers is significant to health care. 


\section{MATERIALS AND METHODS}

Articles published between 2007 and 2013 were collected for this review from Medline, Pub Med and reference lists of selected articles. The MeSH terms used during the search were random hand hygiene audits in acute care settings, hand hygiene in acute care setting, hand hygiene compliance in acute care settings, random safety audits in acute care settings. The initial search returned more than 500 investigations published since 2007. The search was further narrowed by the terms hand hygiene compliance in acute care setting and random audit in acute care setting to reflect the topic of this review. This additional search returned 13 studies about hand hygiene compliance or hand hygiene audits. All 13 studies were selected for review.

\section{RESULTS}

A one-year multicenter collaborative study of product/volume usage measurement and feedback found that hand hygiene compliance occurs at or below $50 \%$ compliance for both intensive care units (26\%) and nonintensive care units (36\%) (McGugkin et al., 2009). After 12 months of measuring product usage and providing feedback by Infection Control Practitioners (ICPs), McGugkin et al. (2009) found that compliance increased to $37 \%$ for ICUs and $51 \%$ for non-ICUs. (ICU, $\mathrm{P}=0.0119$; non-ICU, $\mathrm{p}<0.001)$. In another study, Rosenthal et al. (2009) selected approximately 22 students per year, beginning in 2004, to participate in the Measure to Achieve Patient Safety (MAPS) at the Ronald Reagan UCLA Medical Center. The students were trained in techniques of measuring and observation and in professional behavior. They participated in weekly and monthly meetings with program leadership, received continuing education from the center's patient safety staff and were trained in observational measurement. Hand washing increased from 50 to $93 \%$ (Rosenthal et al., 2009).

Son et al. (2011) implemented a hand-washing program in a medical center between 2008 and 2010, increasing hand washing compliance from 70 to $97 \%$ by training staff members on the World Health Organization (WHO) hand hygiene guidelines. Between 2006 and 2008, the average hand hygiene compliance of the medical cancer center was $65 \%$. After implementation of the new program, the rate increased to $97 \%$; the rate has remained at this level ever since (Son et al., 2011). A study from the United Kingdom that involved 60 hospital units over a 3-year period investigated the effects of feedback intervention on improving hand-hygiene compliance than the United Kingdom's national "Clean your hands" campaign (Pan et al., 2013). The intervention cohort had $18 \%$ increase compliance with hand washing in intensive care units and $13 \%$ on the general acute-care wards.

In a 7-month interventional study that consisted of education about hand hygiene indications and techniques, workplace reminder posters, focused group sessions and feedback on hygiene compliance and infection rates, (Salama et al., 2013) found that the overall hand hygiene compliance rate by all health care workers increased from $42.9 \%$ pre-intervention to $61.4 \%$ post-intervention. Compliance was highest among nurses, with rates from 49.9 to $82.5 \%$ and lowest among doctors, from 38.6 to $43.2 \%$. Remarkable reductions also occurred in the rate of overall healthcare associated infections/1000 patient days from 37.2 to 15.1 , the rate of bloodstream infections from 18.6 to 3.4 central-line days and the rate of lower respiratory tract infections from 17.6 to $5.2 / 1000$ ventilator-days. Similarly, (Salama et al., 2013) found significant reductions in the isolation rates of four major hospital pathogens.

In a study conducted in a 6-bed neurosurgical intensive care unit with technical development and evaluation phases, healthcare workers wore an electronic device in the style of an identity badge to detect hand hygiene opportunities and compliance. Cheng et al. (2011) compared the compliance determined by the system and an infection control nurse. At the same time, the system assessed compliance by time of day, day of week, work shift, professional category of health care providers and individual subject, while the workload of HCWs was monitored by measuring the amount of time they spent in patient zones. Access to hygiene compliance information resulted in an overall compliance increase of $35.1 \%$ for the unit (Cheng et al., 2011).

A pilot study by (Erasmus et al., 2010) explored the practicality and effects of action planning on the hand hygiene behavior of nurses in an ICU and surgical ward of a university teaching hospital used a pre-post test design. Seventeen nurses were invited to participate. A trained researcher observed hand hygiene behavior of nurses before and 3 weeks after the intervention in which action plans were formulated. Hand Hygiene Behavior (HHB) improved from $9.3 \%$ at baseline to $25.4 \%$ post intervention (Erasmus et al., 2010).

A cross-sectional study conducted by direct observation using the Lewisham observation tool and self-administered questionnaire in six major public 
secondary care hospitals in Kuwait resulted in an overall compliance increase of $33.4 \%$ after staff training (Al-Wazzan et al., 2011). Compliance rates significantly varied between different ward categories from 14 in emergency to $55 \%$ in medical wards. Being busy with work $(42.2 \%)$, having sore/dry hands $(30.4 \%)$ and wearing gloves $(20.3 \%)$ were the most frequently reported hindrances to improving hand hygiene in that study. Only patient care activities that are described as 'dirty contacts' by the Fulkerson scale were considered as indications for hand hygiene while any attempt for hand hygiene was considered as compliance (Al-Wazzan et al., 2011).

Garus-Pakowska (2011) employed a quasiparticipation method, comprising all members of medical personnel (186 nurses and physicians) working at six wards of three hospitals, to assess the impact of actual workload on compliance and found that higher work overload increased the frequency of noncompliance with hand hygiene.

Davis (2010) reported 62\% compliance after redecorating the entrance of the ward with a conspicuous strip of bright red tape pointing to alcohol gel dispensers. Compliance improved for all persons entering the ward with doctors improving from 0 to $54 \%$, nurses improving from 24 to $75 \%$, porters improving from 21 to $67 \%$, visitors improving from 35 to $68 \%$ and patients improving from 23 to $44 \%$.

\section{DISCUSSION}

Many studies have documented the importance of hand hygiene compliance by health care workers. The majority of the work conducted to improve hand hygiene compliance has focused on changing the physical environments of healthcare facilities, such as redecorating the entrance of hospital buildings to channel the attention of care-givers and visitors to alcohol gel dispensers and educational and motivational methods, such as online educational programs and staff training. The review of literature indicates that these types of interventions resulted in improvements in compliance rates, ranging from 25.4 to $97 \%$. However, additional studies are needed to evaluate other factors that may improve the hand hygiene compliance of healthcare workers.

Future studies should evaluate the role of random audits combined with feedback on the hand hygiene behavior of health care workers. Random audit is already considered an essential part of clinical practice everywhere. Random audits can be performed and feedback given to health care providers in the morning after the audit. If done in the proper spirit of working together to improve the care of patients, random audits followed by feedback hypothetically will increase hand hygiene compliance, increase awareness of infection control measures, improve infection control practices, provide continued emphasis on infection control and general good clinical practice and improve teamwork. This proposition is supported by several of the studies discussed in this study that demonstrated the potential effect of random audits and feedback on hand hygiene compliance. Such results could translate to huge benefits for the patient whose hospital stay will hopefully be free of infections.

\section{CONCLUSION}

Hand washing is an important means of preventing hospital acquired infections. It is therefore, necessary that hospitals and healthcare facilities explore and implement strategies that have been shown to enhance hand washing compliance. Implementing such strategies could save money and reduce patient sufferings.

\section{REFERENCES}

Al-Wazzan, B., Y. Salmeen, E. Al-Amiri, A. Abul and M. Bouhaimed et al., 2011. Hand hygiene practices among nursing staff in public secondary care hospitals in Kuwait: Self-report and direct observation. Med. Principles Pract., 20: 326-331. PMID: 21576991

CDCP, 2011. Guideline for hand hygiene in healthcare settings. Center of Disease Control and Prevention.

Cheng, V.C.C., J.W. Tai, J.F. Chan, K.H. Sara and W.M.T. Josepha et al., 2011. Introduction of an electronic monitoring system for monitoring compliance with moments 1 and 4 of the WHO "My 5 Moments for hand Hygiene" methodology. BMC Infec. Dis., 26: 151-162. DOI: 10.1186/1471-2334-11-151

Davis, C.R., 2010. Infection-free surgery: How to improve hand-hygiene compliance and eradicate methicillin-resistant Staphylococcus aureus from surgical wards. Ann. R. Coll. Surg. Eng., 92:316169. PMID: 20514722

Erasmus, V., M.N. Kuperus, J.H. Richardus, M.C. Vos and A. Oenema et al., 2010. Improving hand hygiene behavior of nurses using action planning: A pilot study in the intensive care unit and surgical ward. J. Hospital Infec., 76: 161-164. DOI: 10.1016/j.jhin.2010.04.024 
Garus-Pakowska, A., 2011. Workload impact on compliance with hygiene procedures in medical personnel. Med. Pr., 62: 369-376 PMID: 21995106

Graves, N., 2004. Economics of preventing hospital infections. Emerg. Infec. Dis., 10: 561-566. DOI: 10.3201/eid1004.020754

McGugkin, M., R. Waterman and J. Govednik, 2009. Hand hygiene compliance rates in the United States: A one-year multicenter collaborative using product/volume usage measurement and feedback. Am. J. Med. Quality, 24: 205-213. DOI: 10.1177/1062860609332369

Pan, S.C., K.L. Tien, I.C. Hung, Y.J. Lin and W.H. Sheng, 2013. Compliance of health care workers with hand hygiene practices: Independent advantages of overt and covert observers. PLoS ONE, 8: 1-7. DOI: 10.1371/journal.pone.0053746.
Rosenthal, T., M. Erbeznik, T. Padilla, T. Zaroda and D.H. Nguyen et al., 2009. Observation and measurement of hand hygiene and patient identification improve compliance with patient safety practices. Acad. Med., 84: 1705-1712. DOI: 10.1097/ACM.0b013e3181bf6988

Salama, M.F., W.Y. Jamal, S.A. Mousa, K.A. AlAbdulghani and V.O. Rotimi, 2013. The effects of hand hygiene compliance on hospital-acquired infections in an ICU setting in a Kuwaiti teaching hospital. J. Infec. Public Health, 6: 27-34. DOI: 10.1016/j.jiph.2012.09.014

Son, C., T. Chuck, T. Childers, S. Usiak and M. Dowling et al., 2011. Practically speaking: Rethinking hand hygiene improvement programs in health care settings. Am. J. InfecT. Control, 39: 716-724. DOI: 10.1016/j.ajic.2010.12.008 